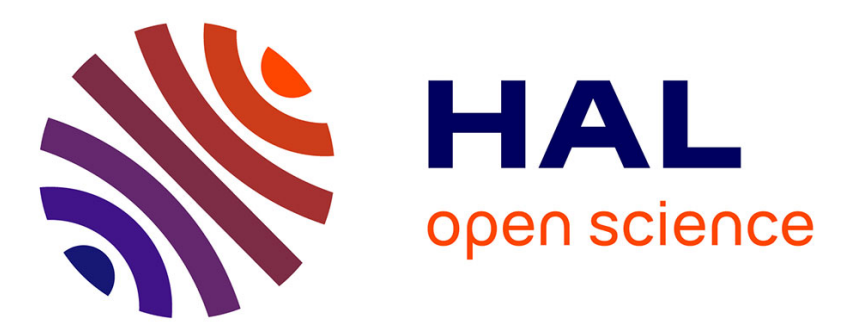

\title{
Adaptive anisotropic mesh technique for coupled problems: application to welding simulation
}

Makhlouf Hamide, Michel Bellet

\section{To cite this version:}

Makhlouf Hamide, Michel Bellet. Adaptive anisotropic mesh technique for coupled problems: application to welding simulation. Materials Processing And Design: Modeling, Simulation and Applications; NUMIFORM '07, Jun 2007, Porto, Portugal. pp.Pages 1561-1566, 10.1063/1.2741031 . hal-00572219

HAL Id: hal-00572219

https://hal-mines-paristech.archives-ouvertes.fr/hal-00572219

Submitted on 11 Mar 2011

HAL is a multi-disciplinary open access archive for the deposit and dissemination of scientific research documents, whether they are published or not. The documents may come from teaching and research institutions in France or abroad, or from public or private research centers.
L'archive ouverte pluridisciplinaire HAL, est destinée au dépôt et à la diffusion de documents scientifiques de niveau recherche, publiés ou non, émanant des établissements d'enseignement et de recherche français ou étrangers, des laboratoires publics ou privés. 


\title{
Adaptive Anisotropic Mesh Technique For Coupled Problems: Application To Welding Simulation
}

\author{
M. Hamide, M. Bellet \\ Ecole des Mines de Paris, Centre de Mise en Forme des Matériaux (CEMEF), UMR CNRS 7635, Sophia \\ Antipolis, France
}

\begin{abstract}
. a major problem arising in finite element analysis of coupled problems, such as welding for instance, is the control of the mesh, that is an appropriate mastering of the spatial discretization to get accurate results in a minimum computer time. The present anisotropic adaptation procedure is controlled by a directional error estimator based on local interpolation error and recovery of the second derivatives of different fields involved in the finite element calculation. Error indicators are derived to define an anisotropic mesh metric field, which is an input of the pre existing 3D remeshing procedure. The mesh metric consists of a combination of several metrics, each corresponding to the error estimation associated with a selected field of the solution produced (temperature, phase fraction, stress component). Mesh modifications are used to anisotropically and continuously adapt the mesh. We demonstrate the efficiency of the method by applying it to a coupled thermal-mechanicalmetallurgical simulation of arc welding. We demonstrate that the use of an anisotropic adaptive finite element method can result in an order of magnitude reduction in computing time with no loss of accuracy compared to analyses obtained with isotropic meshes.
\end{abstract}

Keywords: finite elements, heat transfer, phase transformation, mesh adaptation, anisotropic metric, error estimation, welding

\section{INTRODUCTION}

The accuracy of a numerical solution obtained by the finite element method depends on the spatial discretization of the physical domain. In many physical problems, among them welding, the solution exhibits anisotropic features creating a demand for elements which are aligned with the solution's anisotropy. In realistic cases, the information required to compute the desired solution field to an acceptable level of accuracy is unknown a priori. An efficient approach to overcome this difficulty consists in applying an adaptative procedure in which the errors arising from spatial discretization are controlled within a specified tolerance.

The concentrated heat input that appears in most welding applications requires a refined discretization in the neighbourhood of the molten region below the moving electrodes. This requires some kind of remeshing capability in order to continuously maintain or regenerate a finely discretized region moving with the heat source. The initial work on an automated remeshing strategy for welding applications was performed by Lindgren et al. [1]. Their work included remeshing of a moving region but did not use any error estimation to guide the remeshing scheme and control the accuracy of the solution produced. They prescribed the refinement/coarsening in the input file so that a smaller distance to the source gave smaller elements. Recently, Runnemalm and Hyun [2] proposed an adaptative strategy that evaluates both the thermal and the mechanical error distribution using a Zienkiewicz-Zhu error estimator [3]. It is combined with a hierarchic remeshing strategy using a so-called graded element. In this approach, the directionality of the error estimation is cannot be taken into account, resulting in isotropic adaptative remeshing.

In the present paper, following the approach initiated by D'Azevedo[4], Fortin [5] and Alauzet and Frey [6], we place particular emphasis on the anisotropic mesh adaptation process generated by a directional error estimator based on the recovery of the second derivatives of the different fields involved in the finite element solution. As shown hereafter, this approach allows us to refine the mesh, stretch and orient the elements in such a way that, along the adaptation process, accurate controlled solutions are obtained while keeping the number of unknowns affordably low.

The organization of the paper is as follows. First, we briefly introduce the numerical model that is used to solve and describe the welding process. Then, we present the overall anisotropic mesh adaptation procedure: the anisotropic error estimator, together with the procedures to get the recovered Hessian matrix are described. In this section we discuss the Hessian strategy and review the concept of a mesh metric field. Finally, we apply the different anisotropic adaptative strategies to welding simulations and discuss the results. 


\section{WELDING ANALYSIS}

During welding, the interaction of the heat source and the material leads to rapid heating, melting and the formation of the weld pool. When the heat source moves away, the weld pool cools and solidifies forming the weld joint. Depending on the welded alloys, as the temperature decreases, various solid-state phase transformations may take place resulting in the final microstructure of the weldment. The properties of a weldment, such as strength, ductility, toughness, and corrosion resistance are significantly affected by its microstructure.

In the present work, a so-called staggered approach was used to couple the thermal, mechanical and microstructural analysis, at each time increment, as shown in Fig.1. The thermal field is first calculated followed by the microstructural evolution and finally the mechanical variables are determined.

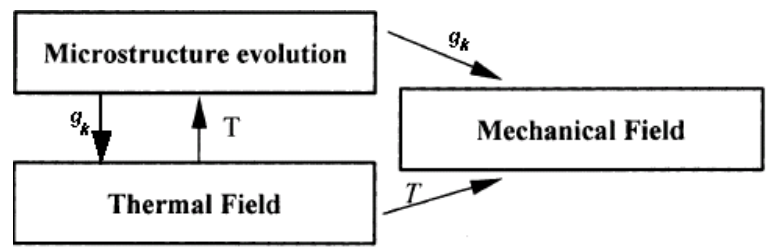

FIGURE 1. Coupling between thermal field, microstructural evolution and mechanical field. $T$ is temperature, $g_{k}$ volume phase fraction of the different phases (index k)

A series of phase transformations take place in both the fusion zone (FZ) and the heat-affected zone (HAZ) during welding of low alloyed steels. The calculation of austenite formation is based on the theory presented by Leblond et al. [7]. The modelling of the transformations during cooling is based on TTT diagrams, extended to non isothermal transformations by using an additivity principle and the notion of fictitious time [8].

The energy conservation in the multiphase material is written using spatial averaging. It is solved for the nodal enthalpies, from which temperature and liquid fraction can be calculated. This formulation takes into account the energy changes associated with the solid state phase changes, while taking advantage of the stability and robustness of the enthalpy formulation for the liquid-solid phase change [9].

The mechanical model uses temperature history data from the thermal model to perform a thermal stress analysis. Along with the thermal stresses, the micro-structure evolution during solid state phase transformation induces some additional stresses. The total strain rate tensor can be written as the sum of the individual components of the strain rate as [10][11] :

$$
\dot{\varepsilon}=\left[\dot{\varepsilon}^{e}+\dot{\varepsilon}^{p}+\dot{\varepsilon}^{t h}\right]+\left[\dot{\varepsilon}^{t r}+\dot{\varepsilon}^{t p}\right]
$$

The various components in this equation represent strain rates due to elastic, plastic and thermal loading, volumetric change and transformation plasticity, respectively. Momentum and mass conservation equations are solved using a velocity-pressure formulation. In the liquid and mushy states, steels are modelled as Newtonian and non Newtonian fluids, respectively, using a temperature dependent viscoplastic model. However, the liquid flow in the weld pool has not been simulated so far, using an arbitrary high viscosity alone the solidus temperature. In the solid state a temperature dependent elasticviscoplastic model is used [11].

\section{MESH ADAPTATION}

In this section, we will briefly present the anisotropic error estimate of the interpolation error based on the second derivatives of the solutions presented since the details can be found in references [6][4][12]

\section{The a posteriori error estimator}

It has been proved that for elliptic problems, the finite element error can be bounded by the interpolation error (Céa's lemma [13]) :

$$
\left\|u-u_{h}\right\| \leq c\left\|u-\Pi_{h} u\right\|
$$

where $\Pi_{h} u$ is the linear interpolate of $u$ on the mesh and $c$ is a constant independent of the current mesh.

Actually, similar analysis based on the interpolation error show (practically) that the link between the interpolation error and the approximation error is even stronger than the bound given by Céa's lemma [13]. Therefore, the interpolation error appears a reasonable way of defining an error estimate according to [5]. Recall that for each element $K$, the anisotropic error interpolation bound involves the second derivatives of the variable $u$ ([6]):

$$
\left\|e_{K}\right\|_{\infty} \leq \operatorname{cmax}_{x \in K} \max _{\vec{e}}\left(\vec{e} \cdot\left|H_{u}(x)\right| \vec{e}\right)
$$

where $\vec{e}$ denotes one of the 6 tetrahedron edges. $c=9 / 32$, $\vec{v}$ is any vector joining two interior points in $K$ and $\left|H_{u}\right|$ is the absolute value of the Hessian matrix of the solution (i.e., consisting of absolute eigenvalues).

The Hessian strategy involves the computation of the symmetric matrix of second derivatives that can be decomposed as $\left|H_{u}\right|=R|\Lambda| R^{T}$, where $R$ is the eigenvectors matrix and $\Lambda=\operatorname{diag}\left(\lambda_{k}\right)$ is the matrix of eigenvalues.

\section{Metric definition}

The stated goal of the mesh adaptation algorithm is to yield a mesh with regular elements in the metric space 
where each edge $e$ must satisfy the following relation (see, for example, [14]):

$$
(\vec{e} \cdot \bar{M} \vec{e})=1
$$

A mesh with all its edges satisfying the above relationship is commonly referred as a unit mesh.

To achieve a suitable mesh resolution in different directions, a uniform distribution of local errors is applied in the principal directions which leads to $c h_{k}^{2} \bar{\lambda}_{k}=\varepsilon$, where $\varepsilon$ is the user specified tolerance for the error and $h_{k}$ is the desired size in the $k_{t h}$ principal direction. So, the edges $\vec{e}$ of the adapted mesh must check $\varepsilon=c(\vec{e} \cdot M \vec{e})$. A mesh metric tensor $\bar{M}$ is then obtained at each node by calculating a scaled eigenspace of the recovered Hessian matrix as $\bar{M}=R \bar{\Lambda} R^{T}$, where $R$ is the eigenvector matrix and $\bar{\Lambda}=\frac{c}{\varepsilon} \Lambda$ is the diagonal matrix of scaled eigenvalues.

When several variables fields $u$ are considered concurrently, the previous approach leads to a metric for each variable and we chose to take the intersection of the different metrics. In practice the intersection of metrics is achieved by the simultaneous reduction of two quadratic forms which is a valid operation since the metric tensors are positive definite [6].

\section{Hessian evaluation}

To compute the Hessian matrix $H_{u}$ of the $P 1$ field $u$, we reconstruct in two steps the second derivatives at each node $P$ by using the computed solution from the patch $S$ of all elements $K$ surrounding node $P$. This is done as follows:

In a first step we recover the gradient at node $P$ by taking the volume weighted average of gradients on elements in the patch $S$. Note that $u$ being a $P 1$ field, its gradient is constant on each element $K$ :

$$
\Pi_{h}\left(\nabla u_{h}\right)(P)=\frac{1}{\sum_{K \in S}|K|}\left(\sum_{K \in S}|K|\left(\nabla u_{h}\right)_{\mid K}\right)
$$

It can also be noticed that this is equivalent to a lumped-mass approximation of a least squares reconstruction of the gradient for linear elements. In a second step, the same procedure is now applied to the $P 1$ field $\Pi_{h}\left(\nabla u_{h}\right)$ to obtain the recovered Hessian matrix.

\section{APPLICATION TO WELDING SIMULATION}

\section{Welding conditions and material properties}

We consider a thick plate of A508 steel, the dimensions of which are given by Fig. 2(a). The temperature dependent thermophysical properties are given in Fig. 3.

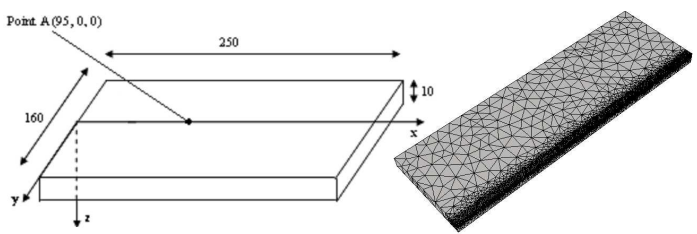

FIGURE 2. a) Geometry of the specimen (dimensions in $\mathrm{mm}$ ) and comparison point A, b) Reference FEM mesh of one half of the plate
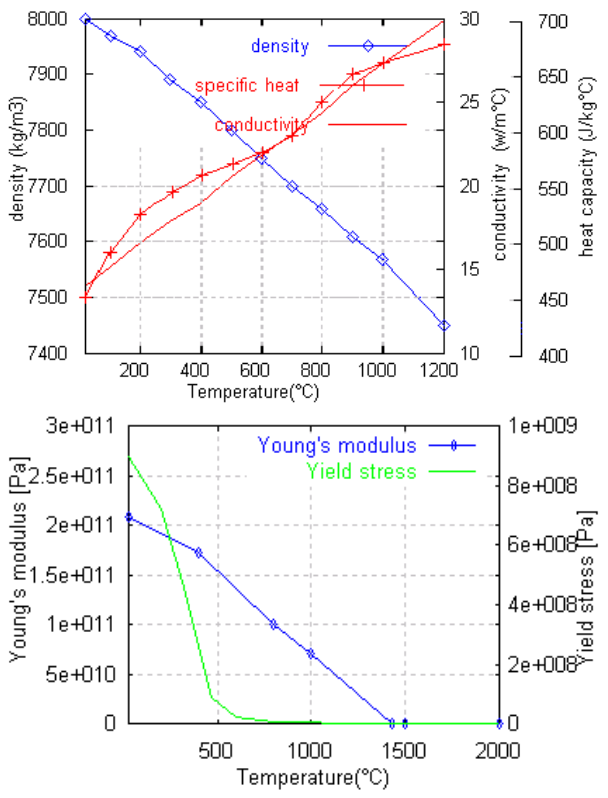

FIGURE 3. Thermal and mechanical material properties used in analysis (SI units)

The welding parameters chosen for this analysis are as follows. Welding process: gas tungsten-arc welding (GTAW); welding current $I=150 \mathrm{~A}$, welding voltage $V=10 \mathrm{~V}$ and travelling speed of $1 \mathrm{~mm} \cdot \mathrm{s}^{-1}$. The weld efficiency is assumed to be $\chi=0.65$. The associated heat input, $I \times V \times \chi$, moving with the electrode, is simulated by a simple surfacic uniform distribution within a disc of radius $5 \mathrm{~mm}$.

\section{Finite element model}

For the simulation study, only one-half of the plate is analyzed due to symmetry. The boundary conditions of the welding process incorporate heat transfer boundary conditions. The symmetry surface is defined as under adiabatic boundary conditions. On all other surfaces boundary conditions of convection and radiation with the environment are applied with a convective coefficient $h=12 \mathrm{Wm}^{-2} \mathrm{~K}^{-1}$ an emissivity coefficient $\varepsilon=0.75$ and an external temperature $T_{\text {ext }}=25 / C$. 
To evaluate the efficiency of our adaptative procedure we first obtain results on a fine mesh (Fig. 2(b)). The result obtained is then used for purpose of comparison. Several simulations have been performed with adaptative remeshing. The calculations differ by the prescribed tolerance error, all other conditions being identical. Three types of simulation with remeshing have been carried out:

- Thermal-driven mesh adaptation : the adaptative technique is based on the thermal error distribution

- Thermometallurgical-driven mesh adaptation : the automatic mesh refinement is based on both thermal and metallurgical error distributions

- Thermomechanical-driven mesh adaptation : the automatic mesh refinement is based on temperature, longitudinal and transversal stresses.

\section{Thermal-based mesh adaptation}

The reference FE-model without remeshing consists of 14329 nodes and 68891 linear tetrahedral elements and is presented in Fig. 2. The initial FE-model used in calculations with remeshing consists of 6842 tetrahedral elements (1683 nodes).

TABLE 1. Refinement parameters (Nbe: denotes the number of elements. Calculation run on a Pentium $4 \mathrm{PC}, 2 \mathrm{GHz}$ with $2 \mathrm{~Gb}$ RAM.

\begin{tabular}{|l|l|l|l|}
\hline & $\begin{array}{l}\text { Initial } \\
\text { Nbe }\end{array}$ & $\begin{array}{l}\text { Final } \\
\text { Nbe }\end{array}$ & $\begin{array}{l}\text { CPU- } \\
\text { Time }\end{array}$ \\
\hline Fine ref. & 68891 & 68891 & $6 \mathrm{~h} 25 \mathrm{~min}$ \\
\hline Coarse ref. & 11439 & 11439 & $58 \mathrm{~min}$ \\
\hline Adapt. $\varepsilon=0.01$ & 6842 & 5866 & $1 \mathrm{~h} 1 \mathrm{~min}$ \\
\hline Adapt. $\varepsilon=0.005$ & 6842 & 11012 & $1 \mathrm{~h} 52 \mathrm{~min}$ \\
\hline
\end{tabular}

The remeshing is performed at each time step $(d t=$ $1 s$ ). See Figs. 2(b) and 4(a) for the FE-mesh. As expected, the adaptative remeshing generates more refined elements in the neighbourhood of the thermal source and coarser elements in its trail. It can be seen also in Fig.4 that anisotropic elements aligned with the heat flow are created around the fusion zone.

As shown in Tab. 1, the calculation on the fine reference mesh (without remeshing) required $6 \mathrm{~h}$ and $25 \mathrm{~min}$ of CPU-time. Two calculations with remeshing have been performed, one with a prescribed error $\varepsilon=0.01$ and a second one with $\varepsilon=0.005$. The CPU-time was $1 \mathrm{~h}$ and $1 \mathrm{~min}$ for $\varepsilon=0.01$ and $1 \mathrm{~h}$ and $52 \mathrm{~min}$ for $\varepsilon=0.005$. As expected, the calculation with $\varepsilon=0.005$ generates a larger refined zone in the neighbourhood of the thermal source than the calculation with $\varepsilon=0.01$.

The temperature evolutions at a given point A (Fig. 2)in the different analyses are shown in Fig. 5. The

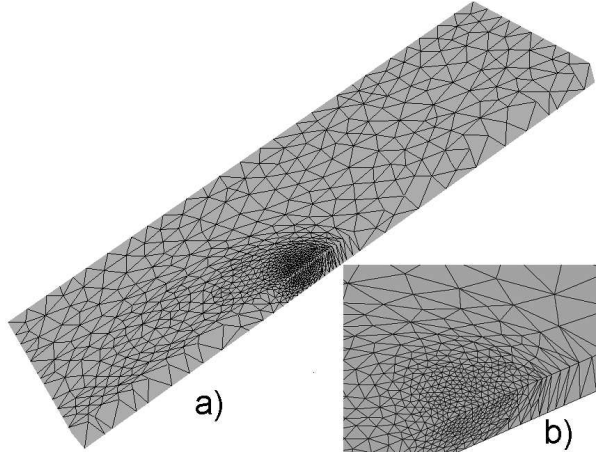

FIGURE 4. Thermal-based mesh adaptation $(\varepsilon=0.01)$, a) Adapted FEM mesh b) Zoom on refined zone

first observation from the plots in Fig. 5 is that the results are significantly smoother in the time domain. This illustrates that the spatial noise associated with the Hessian recovery does not globally pollute the solution in time, suggesting that the primary fields (temperature and phase fractions) remain unaffected. We observe in Fig. 5 that the temperature evolution à Point A converges to the reference temperature evolution when reducing the prescribed error $\varepsilon$. From Tab. 1 and Fig. 5, it can be seen that for a comparable accuracy of the results, the use of an adaptative meshing procedure reduces CPU costs by a factor six, which demonstrates the efficiency of the proposed approach.

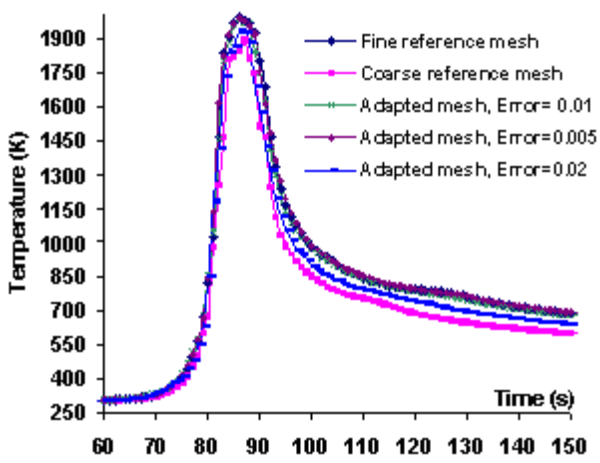

FIGURE 5. Thermal-based mesh adaptation $(\varepsilon=0.01)$, Temperature evolution at Point A (2), [K]

\section{Thermo-metallurgical based mesh adaptation}

Comparing Fig.4 and Fig.6 a clear difference between the obtained two meshes when using only the thermal error distribution Fig. 4 or both the thermal and the metallurgical error distributions is evidenced. A distinct behaviour is found when guiding the mesh adaptation with both phase proportion (in the present case, 


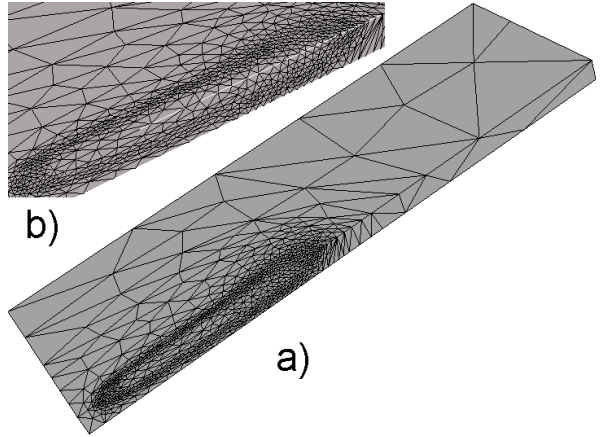

FIGURE 6. Thermo-metallurgical based mesh adaptation $(\varepsilon=0.01)$, a) Adapted FEM mesh, b) Zoom on refined zone

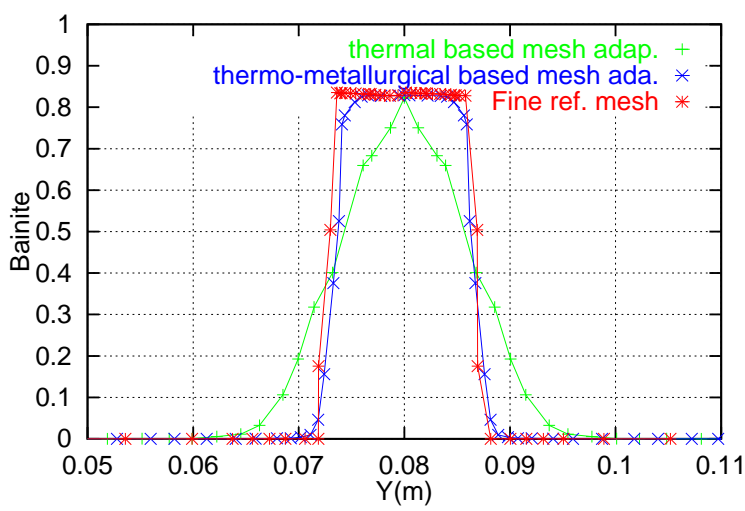

FIGURE 7. Profiles of bainite volume fraction in a crosssection located at $X=0.095 \mathrm{~m}$ at time $250 \mathrm{~s}$

the bainite volume fraction) and temperature. In this case, the mesh is much denser in the wake of the heat source in order to provide a better representation of the steep gradients of phase fraction. It can be seen that the thermometallurgical-driven mesh generation produces significantly more elements than the thermal-driven one (see Tab. 2, Figs. 4 and 6). This is due to the residual gradient of phases fractions that remains in the welded component after welding.

TABLE 2. Refinement parameters and results for thermalmetallurgical adaptation. Calculation run on a Pentium 4 PC, $2 \mathrm{GHz}$ with $2 \mathrm{~Gb}$ RAM.

\begin{tabular}{|l|l|l|l|}
\hline & Initial Nbe & Final Nbe & CPU-Time \\
\hline Fine ref. & 68891 & 68891 & 6h 25min \\
\hline Adapt. $\varepsilon=0.01$ & 6842 & 15816 & 2h 22min \\
\hline
\end{tabular}

Fig. 7 shows the residual profile of the bainite volume fraction in a transverse section, using different meshes. It can be seen that the phase distribution is much more accurate than with an adaptation based only on the thermal error distribution.

\section{Thermomechanical based mesh adaptation}

Mechanical properties applied in the thermomechanical model are shown in Fig.3. In order to simplify the analysis, we don't take into account the effect of metallurgical transformation here. The conditions of thermal calculation are unchanged. As to mechanical boundary conditions when welding, the plate has been fixed at both lateral faces (parallel to the welding direction). The symmetric simplification is not considered, the whole plate has been modelled. The mesh adaptation is based on three fields: temperature, longitudinal and transversal stresses.

The reference mesh and the mesh refinement obtained by using temperature and stress as error indication are shown in Fig. 8. A distinct behavior is found when using a purely thermal mesh adaptation (Fig.4) which gives much lower number of elements in the model. The evolution of the calculated longitudinal and transverse stresses is shown in Fig. 8 to 10, it can be seen that the simulation using remeshing gives similar results to the simulation on reference mesh. The reduction in size of the models decreases the required CPU-time by almost one-third (Tab.3). Figure 10 shows that the stress change during welding is complex and depends on geometry and constraint. Thus the thermal-mechanical adaptation is necessary when modelling complex three-dimensional welding problems as even an experienced user may find it difficult to mesh the model efficiently.

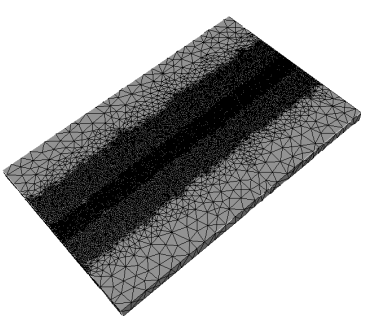

a)

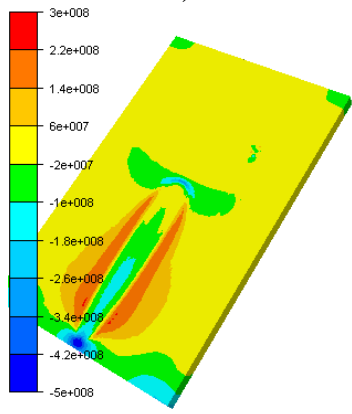

c)

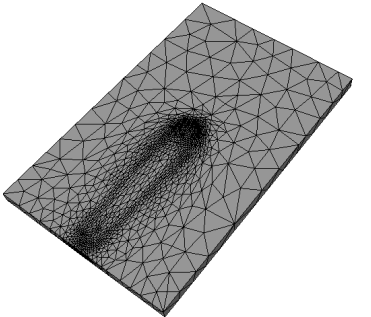

b)

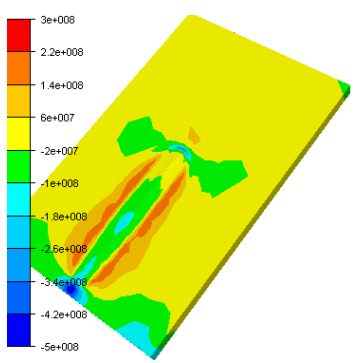

d)
FIGURE 8. a) Reference mesh, b) adapted mesh based on temperature and stress; Transverse stress (in $\mathrm{Pa}$ ) on reference mesh (c) and on adapted mesh (d) 


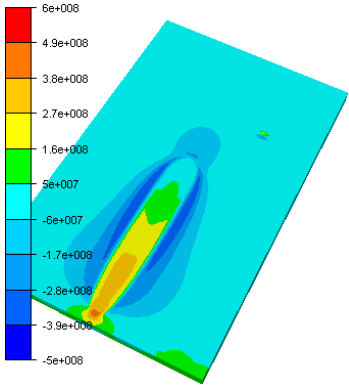

a)

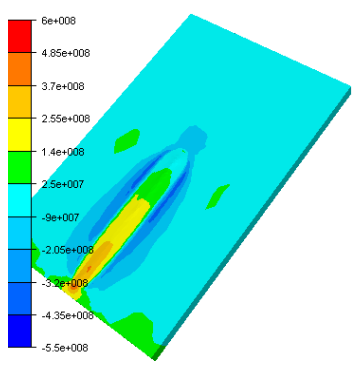

b)
FIGURE 9. Longitudinal stress (in Pa) on reference mesh (a) and on adapted mesh (b)

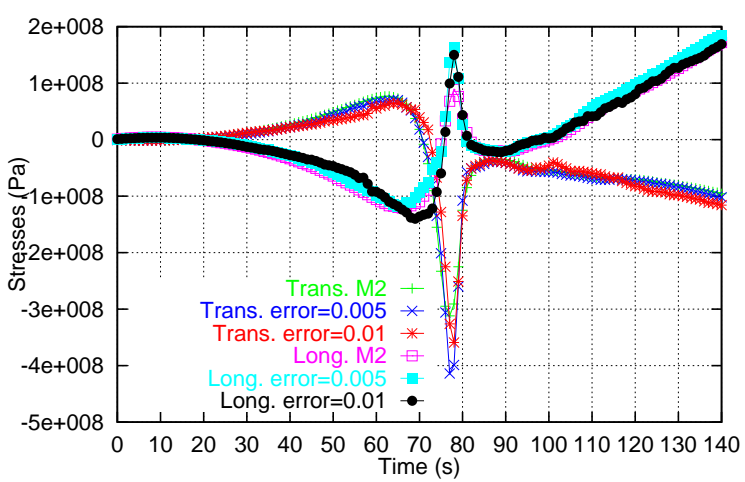

FIGURE 10. Longitudinal and Transverse stresses profiles (in Pa) obtained on different meshes

TABLE 3. Refinement parameters and results for thermalmetallurgical adaptation. Calculation run on a Pentium 4 PC, $2 \mathrm{GHz}$ with $512 \mathrm{Mb}$ RAM.

\begin{tabular}{|l|l|l|l|}
\hline & $\begin{array}{l}\text { Initial } \\
\text { Nbe }\end{array}$ & $\begin{array}{l}\text { Final } \\
\text { Nbe }\end{array}$ & CPU-Time \\
\hline Fine ref. mesh & 194263 & 194263 & $81 \mathrm{~h} 07 \mathrm{~min}$ \\
\hline Adapt. $\varepsilon=0.005$ & 7152 & 27447 & $26 \mathrm{~h} 10 \mathrm{~min}$ \\
\hline
\end{tabular}

\section{CONCLUSION}

In this paper, adaptive remeshing procedures have been presented and applied in the context of coupled thermalmechanical-metallurgical simulations of welding. The method is based on anisotropic mesh adaptivity dictated by directional error estimators. Those estimators, based on the Hessian recovery of $P 1$ field, are used to construct a mesh metric field that provides information on the local mesh resolution desired in different directions. The method allows to easily combine metric tensors for variables of different type and nature. In practice, the calculation results show that the temperature field, the distributions of phase fractions and stress obtained with adaptive mesh converge to the results obtained with reference meshes. For the cases tested here, the calculation time comparison shows that the adaptive mesh technique can reduce the calculation time by almost one-third. It also reduces the data-storage requirement substantially. For some applications, both points are key-factors in determining whether a successful FE-simulation can be completed or not.

\section{REFERENCES}

1. L.E. Lindgren, H.A. Haggblad, J.M.J. McDill, and A.S. Oddy. Automatic remeshing for three-dimensional finite element simulation of welding. Comp. Meth. Appl. Mech. Eng., (147):401-409, 1997.

2. H. Runnemalm and S. Hyun. Three-dimensional welding analysis using an adaptive mesh scheme. Comput. Methods Appl. Mech. Engrg., (189):515-523, 2000.

3. O.C. Zienkiewicz and J.Z. Zhu. A simple error estimator and adaptive procedure for practical engineering analysis. Int. J. Numer. Meth. Eng., (24):337-357, 1987.

4. E.F. D'Azevedo and B. Simpson. On optimal triangular meshes for minimizing the gradient error. Numerische Mathematik, (59(4)):321-348, 1991.

5. M. Fortin. Estimation d'erreur a posteriori et adaptation de maillages. Revue européenne des éléments finis, 9(4):467-486, 2000.

6. F. Alauzet, P.J. Frey, and P.L. George. Anisotropic mesh adaptation for rayleigh-taylor instabilities. European Congress on Computational Methods in Applied Sciences and Engineering (ECCOMAS), 2004.

7. J.B Leblond, J. Devaux, and J.C. Devaux. A new kinetic model for anisothermal metallurgical transformations in steels including effect of austenite grain size. Acta Metall, (32(1)), 1959.

8. S. Denis and D. Farias. Mathematical model coupling phase transformations and temperature evolutions in steels. ISIJ Int., (32):316-325, 1992.

9. M. Hamide, E. Massoni, and M. Bellet. Adaptive mesh technique for thermal-metallurgical numerical simulation of arc welding processes. submitted to Int. J. Num. Meth. Engng., 2006.

10. C. Aliaga. Simulation numérique par éléments finis en $3 D$ du comportement thermomécanique au cours $d u$ traitement thermique d'acier : application à la trempe de pièces forgées ou coulées. Thèse de doctorat, Ecole des mines de Paris, (France), 2000.

11. M. Bellet, O. Jaouen, and I. Poitrault. An ale-fem approch to the thermomechanics of solidification processes with application to the prediction of pipe shrinkage. Int. J. Num. Meth. Heat Fluid Flow, 15(2):120-142, 2005.

12. N.P. Garcia M.V. Anglada and P.B. Crosa. Directional adaptive surface triangulation. Comput. Aided Des., (16):107-126, 1999.

13. P.G. Ciarlet. Basic error estimates for elliptic problems. In North Holland Eds, editor, Handbook of Numerical Analysis, volume 2, chapter Finite Element methods, pages 17-352. P.G. Ciarlet and J.L. Lions, 1991.

14. C. Gruau and T. Coupez. $3 \mathrm{~d}$ tetrahedal unstructured and anisotropic mesh generation with adaptation to natural and multidomain metric. Comput. Methods Appl. Mech. Engrg., 194:4951-4976, 2005. 